\title{
Levels of S100 calcium binding protein B (S100B), neuron-specific enolase (NSE), and cyclophilin A (CypA) in the serum of patients with severe craniocerebral injury and multiple injuries combined with delirium transferred from the ICU and their prognostic value
}

\author{
Yang Gao ${ }^{1,2 \#}$, Jun Duan ${ }^{2,3 \#}$, Hong $\mathrm{Ji}^{2,4}$, Weifeng $\mathrm{Lu}^{2,5}$ \\ ${ }^{1}$ Department of general practice center, Sichuan Provincial People's Hospital, University of Electronic Science and Technology of China, Chengdu, \\ China; ${ }^{2}$ Chinese Academy of Sciences Sichuan Translational Medicine Research Hospital, Chengdu, China; ${ }^{3}$ Department of Geriatrics, Sichuan \\ Provincial People's Hospital, University of Electronic Science and Technology of China, Chengdu, China; ${ }^{4}$ Department of geriatric medicine, \\ Sichuan Provincial People's Hospital, University of Electronic Science and Technology of China, Chengdu, China; ${ }^{5}$ Department of anesthesia \\ operation center, Sichuan Provincial People's Hospital, University of Electronic Science and Technology of China, Chengdu, China \\ Contributions: (I) Conception and design: Y Gao, J Duan; (II) Administrative support: H Ji, W Lu; (III) Provision of study materials or patients: Y \\ Gao, J Duan; (IV) Collection and assembly of data: Y Gao, J Duan; (V) Data analysis and interpretation: Y Gao, J Duan; (VI) Manuscript writing: All \\ authors; (VII) Final approval of manuscript: All authors. \\ "These authors contributed equally to this work. \\ Correspondence to: Weifeng Lu. Department of Anesthesia Operation Center, No.32 West Section 2, First Ring Road, Chengdu 610072, China. \\ Email: fengjie667@163.com; Hong Ji. Department of Geriatric Medicine, No.32 West Section 2, First Ring Road, Chengdu 610072, China. \\ Email: ekkjxstmimwh30@sina.com.
}

Background: To analyze the levels of S100 calcium binding protein B (S100B), neuron-specific enolase (NSE), and cyclophilin A (CypA) in the serum of patients with severe craniocerebral injury combined with delirium and multiple injuries transferred from the intensive care unit (ICU), and their prognostic value.

Methods: The data of 98 patients with severe craniocerebral injury combined with delirium and multiple injuries admitted to our hospital from January 2018 to May 2019 were retrospectively analyzed as the study group. The differences in serum S100B, NSE, and CypA levels in each group were compared, and the deaths of the study group during follow-up were counted.

Results: The levels of S100B, NSE, and CypA in the study group were higher than those in the control group $(\mathrm{P}<0.05)$. The mortality rate of the 98 patients with severe craniocerebral injury combined with delirium and multiple injuries transferred from the ICU was 37.76\%. Furthermore, the levels of S100B, NSE, and CypA in the death group were higher than those in the survival group $(\mathrm{P}<0.05)$. Glasgow Coma Score (GCS) score $\leq 5$ points, Injury Severity Score (ISS) score $>25$ points, multiple organ dysfunction syndrome, and increased levels of S100B, NSE, and CypA were independent risk factors that affected the prognosis of patients with severe craniocerebral injury combined with delirium and multiple injuries transferred from the ICU $(\mathrm{P}<0.05)$. The average survival times of the high S100B level group, the high NSE level group, and the high CypA level group were shorter than those of the low-level groups $(\mathrm{P}<0.05)$.

Conclusions: The levels of S100B, NSE, and CypA in serum were closely related to the prognosis of patients with severe craniocerebral injury combined with delirium and multiple injuries transferred from the ICU. They can be used as molecular markers for predicting the prognosis of patients, and may serve as potential targets for treatment.

Keywords: Severe craniocerebral injury-based multiple injuries; delirium; serum S100 calcium binding protein B (serum S100B); neuron-specific enolase (NSE); cyclophilin A (CypA) 
Submitted Jan 28, 2021. Accepted for publication Mar 16, 2021.

doi: 10.21037/apm-21-424

View this article at: http://dx.doi.org/10.21037/apm-21-424

\section{Introduction}

Multiple injuries refer to the same injury factor causing 2 or more serious injuries to the human body at the same time or one after another. In recent years, the incidence of multiple injuries has increased year by year. Among them, severe head injuries combined with multiple injuries account for $10 \%$ to $20 \%$ (1). Delirium is a relatively common clinical acute mental syndrome with rapid clinical development. During the period of admission to the intensive care unit (ICU) for severe head injury, patients with multiple injuries are more likely to experience delirium than ordinary patients, under the combined influence of environmental factors, psychological factors, therapeutic measures, and disease $(2,3)$. With the development of intensive care medicine, the initial treatment success rate of patients with severe head injuries and delirium combined with multiple injuries has been greatly improved. In recent years, the prognosis of patients transferred from the ICU has become the focus of attention $(4,5)$.

S100 calcium binding protein B (S100B) is a specific index that can predict the severity of cerebrovascular damage, guide clinical treatment, and judge prognosis. Neuron-specific enolase (NSE) is a specific marker of neuronal damage. The value of both markers in craniocerebral injuries has been recognized $(6,7)$. Cyclophilin A (CypA) is an important factor involved in immune regulation, inflammatory responses, and cell growth, amongst other processes, and its role in the prognostic evaluation of critically ill patients has attracted attention (8). Increased serum CypA concentrations reflect trauma severity and unfavorable outcomes after head trauma, making CypA a potential biomarker for the prognostic prediction of traumatic brain injury (TBI) (9). This study analyzed the serum levels of S100B, NSE, and CypA in patients transferred from the ICU with severe head injury and delirium, aiming to reveal the value of these 3 markers for the prediction of prognosis. We present the following article in accordance with the STROBE reporting checklist (available at http://dx.doi. org/10.21037/apm-21-424).

\section{Methods}

\section{Patient information}

In this retrospective analysis, 98 patients with combined severe craniocerebral injury and delirium from January 2018 to May 2019 at our hospital were used as the research group. The study was conducted in accordance with the Declaration of Helsinki (as revised in 2013). This study was approved by the Sichuan Provincial People's Hospital, University of Electronic Science and Technology of China (No. 20171208). All patients agreed to participate in this study and signed an informed consent form. The inclusion criteria were as follows: (I) a clear history of head injury; (II) Glasgow Coma Score (GCS) (10) $\leq 8$ points; (III) head CT examination after admission showed brain contusion or brain injury contusion and laceration, obvious intracerebral hematoma and/or brain herniation, brain stem injury, and/ or multiple skull fractures; (IV) Injury Severity Score (ISS) (11) $\geq 16$ points; (V) the clinical data was complete with no missing information. The exclusion criteria were as follows: (I) patients had concomitant serious organic diseases of the heart, liver, and kidney, or other tumors; (II) occurrence of heart, cerebral stroke, or brain trauma within the 6 months before enrollment; (III) use of anticoagulants or anti-platelet drugs within the 3 months before enrollment; (IV) had blood system diseases, such as combined leukemia, idiopathic thrombocytopenic purpura, or hemophilia, amongst others; (V) had acute and chronic infections within the 1 month before enrollment.

There were 53 males and 45 females in the research group, aged from 18 to 70 years old, with an average age of $39.64 \pm 8.57$ years old. Among them, 46 were traffic accidents, 22 were bruises, 20 were fall injuries, and the other 10 were. There were 76 cases of closed head injury and 22 cases of open head injury. The control group consisted of 90 patients who had a physical examination during the same period in our hospital. There were no statistically significant differences in age and gender compared with the research group, and they were comparable $(\mathrm{P}>0.05)$. 
Table 1 Comparison of S100B, NSE, and CypA levels between the research group and the control group

\begin{tabular}{lccc}
\hline Group & S100B $(\mathrm{pg} / \mathrm{mL})$ & $\mathrm{NSE}(\mu \mathrm{g} / \mathrm{L})$ & CypA $(\mathrm{ng} / \mathrm{mL})$ \\
\hline $\begin{array}{l}\text { Research group } \\
(\mathrm{n}=98)\end{array}$ & $624.51 \pm 120.36$ & $39.62 \pm 12.74$ & $99.63 \pm 30.41$ \\
$\begin{array}{l}\text { Control group } \\
(\mathrm{n}=90)\end{array}$ & $36.74 \pm 10.59$ & $10.50 \pm 2.31$ & $13.54 \pm 3.48$ \\
$\mathrm{t}$ & 46.155 & 21.360 & 26.691 \\
P value & $<0.001$ & $<0.001$ & $<0.001$ \\
\hline
\end{tabular}

S100B, S100 calcium binding protein B; NSE, neuron-specific enolase; CypA, cyclophilin A.

\section{Research methods}

After admission, the patients in the study group were diagnosed based on the results of medical history, physical examination, laboratory examination, and special examinations (such as B-ultrasound, chest X-ray, CT, etc.), and the injury was fully estimated. The combined injuries were then treated in the order of chest, abdomen, brain, and limbs. That is, for patients with thoracic organ damage and hemorrhagic shock, controlling the bleeding and correcting dyspnea were the first tasks, followed by strengthening the treatment and support for the damage of vital organs, such as maintaining airway patency, anti-shock therapy, handling blood pneumothorax. If there were multiple organ injuries in the abdominal cavity, the first task was to control bleeding and repair the perforated organ as soon as possible, and remove the intracranial hematoma at the same time or later. Both postoperative patients and non-surgical patients received conventional trauma surgery interventions, dehydrating agents, brain protective agents, prevention of infection, enteral nutrition support, or rehabilitation treatment as needed.

The clinical data of all the study subjects were collected, and the serum levels of S100B, NSE, and CypA were compared between the research group (within 12 hours before being transferred from the ICU) and the control group. Subsequently, 98 patients who were transferred out of the ICU with severe head injury and delirium were followed up for a period of 30 days, and their deaths during the follow-up period were counted. The related factors that affected the prognosis of patients transferred from the ICU with severe head injury and delirium were analyzed by unconditional univariate and multivariate logistic regression models. Kaplan-Meier survival curves were generated to study the effects of serum S100B, NSE, and CypA levels on the prognosis of patients.

\section{Determination of S100B, NSE, and CypA levels}

Venous blood (cubital vein) was collected in a heparin anticoagulation tube and centrifuged $(1,500 \mathrm{r} / \mathrm{min}$, $10 \mathrm{~min}$ ), and the supernatant was stored in a refrigerator at $-20{ }^{\circ} \mathrm{C}$. The serum levels of NSE, S100B, and CypA were determined by enzyme-linked immunosorbent assay (ELISA). The Elecsys2010 electroluminescence automatic immunoassay instrument (Roche, Japan) was use for detection, and the NSE kit was produced by Roche, Germany. The serum levels of S100B and CypA were detected using the Bio-Rad Coda automatic enzyme immunoassay instrument (Bio-Rad, Hercules, CA, USA) according to the kit instructions.

\section{Statistical analysis}

The data in this study were statistically analyzed by SPSS22.0 software, the count data were expressed as n (\%), and an independent sample t-test was used. Multivariate logistic regression analysis was used to analyze the risk factors affecting the prognosis and survival of patients transferred from the ICU with severe head injury and delirium. Kaplan-Meier survival curves were used to assess the predictive value of S100B, NSE, and CypA for the prognosis of patients. Differences were statistically significant when $\mathrm{P}<0.05$.

\section{Results}

\section{Comparison of S100B, NSE, and CypA levels between the} research group and the control group

The expression levels of S100B, NSE, and CypA in the research group were higher than those in the control group $(\mathrm{P}<0.05)$, as shown in Table 1.

\section{Prognosis and survival of the 98 patients}

By the end of the follow-up, out of the 98 patients who were transferred out of the ICU with severe head injury and delirium, 37 patients died, 61 patients survived, and the mortality rate was $37.76 \%$. 
Comparison of S100B, NSE, and CypA levels between the survival group and death group

The expression levels of S100B, NSE, and CypA in the death group were higher than those in the survival group $(\mathrm{P}<0.05)$, as shown in Table 2.

\section{Multivariate analysis}

GCS score $\leq 5$ points, ISS score $>25$ points, multiple organ

Table 2 Comparison ofS100B, NSE, and CypA levels between the survival group and death group

\begin{tabular}{lccc}
\hline Group & $\mathrm{S} 100 \mathrm{~B}(\mathrm{pg} / \mathrm{mL})$ & $\mathrm{NSE}(\mu \mathrm{g} / \mathrm{L})$ & CypA $(\mathrm{ng} / \mathrm{mL})$ \\
\hline $\begin{array}{l}\text { Death group } \\
(\mathrm{n}=37)\end{array}$ & $706.41 \pm 50.74$ & $49.86 \pm 3.67$ & $110.80 \pm 10.59$ \\
$\begin{array}{l}\text { Survival group } \\
(\mathrm{n}=61)\end{array}$ & $563.05 \pm 52.95$ & $20.71 \pm 3.85$ & $85.69 \pm 12.03$ \\
$\mathrm{t}$ & 13.197 & 36.974 & 10.468 \\
$\mathrm{P}$ value & $<0.001$ & $<0.001$ & $<0.001$ \\
\hline
\end{tabular}

S100B, S100 calcium binding protein B; NSE, neuron-specific enolase; CypA, cyclophilin A; P value, Probability dysfunction syndrome, and abnormally elevated levels of S100B, NSE, and CypA were independent risk factors for the prognosis of patients with multiple injuries combined with delirium who were transferred out of the ICU with severe head injury $(\mathrm{P}<0.05)$, as shown in Table 3.

\section{The relationship between S100B, NSE, and CypA levels on the prognosis and survival of patients}

As shown in Figure 1, Kaplan-Meier survival curves were significantly different between the different expression levels of S100B, NSE, and CypA. The average survival time of the high S100B level group was shorter than that of the low-level group $(\mathrm{P}<0.05)$. The average survival time of the high NSE level group was shorter than that of the low-level group $(\mathrm{P}<0.05)$. The average survival time of the high CypAlevel group was shorter than that of the low-level group $(\mathrm{P}<0.05)$.

\section{Discussion}

Multiple injuries combined with craniocerebral injury are harmful, and are one of the most significant causes

Table 3 Multivariate analysis of factors affecting the prognosis of patients transferred out of the ICU with severe head injury and delirium combined with multiple injuries

\begin{tabular}{|c|c|c|c|c|c|c|}
\hline Variable & \multicolumn{3}{|c|}{ Univariate analysis } & \multicolumn{3}{|c|}{ Multivariate analysis } \\
\hline Age (<60 vs. $\geq 60$ years) & 2.342 & $1.805-3.039$ & 0.025 & 1.642 & $0.814-3.312$ & - \\
\hline Gender (male vs. female) & 2.759 & $0.917-8.302$ & 0.162 & - & - & - \\
\hline GCS score ( $>5$ vs. $\leq 5$ points) & 2.333 & $1.611-3.379$ & 0.006 & 4.669 & $1.182-18.448$ & $<0.001$ \\
\hline High blood sugar (yes vs. no) & 4.531 & $3.129-6.563$ & 0.007 & 2.396 & $0.531-10.818$ & 0.094 \\
\hline Shock (yes vs. no) & 2.601 & $2.04-3.376$ & 0.019 & 2.883 & $0.422-19.684$ & \\
\hline Multiple organ dysfunction syndrome (yes vs. no) & 2.396 & $1.655-3.471$ & $<0.001$ & 2.433 & $1.487-3.979$ & $<0.001$ \\
\hline Systemic inflammatory response syndrome (yes vs. no) & 2.604 & $1.798-3.771$ & $<0.001$ & 2.649 & $0.670-10.464$ & 0.130 \\
\hline S100B levels (elevated vs. normal) & 2.349 & $1.914-3.043$ & $<0.001$ & 2.625 & $1.605-4.293$ & $<0.001$ \\
\hline NSE levels (elevated vs. normal) & 4.116 & $1.368-12.385$ & $<0.001$ & 2.591 & $1.584-4.237$ & $<0.001$ \\
\hline CypA levels (elevated vs. normal) & 2.787 & $2.181-3.561$ & $<0.001$ & 2.875 & $1.758-4.702$ & $<0.001$ \\
\hline
\end{tabular}

ICU, intensive care unit; GCS, Glasgow Coma Score; ISS, Injury Severity Score; S100B, S100 calcium binding protein B; NSE, neuronspecific enolase; CypA, cyclophilin A. OR, odds ratio; $\mathrm{Cl}$, Confidence interval; P value, probability 
A

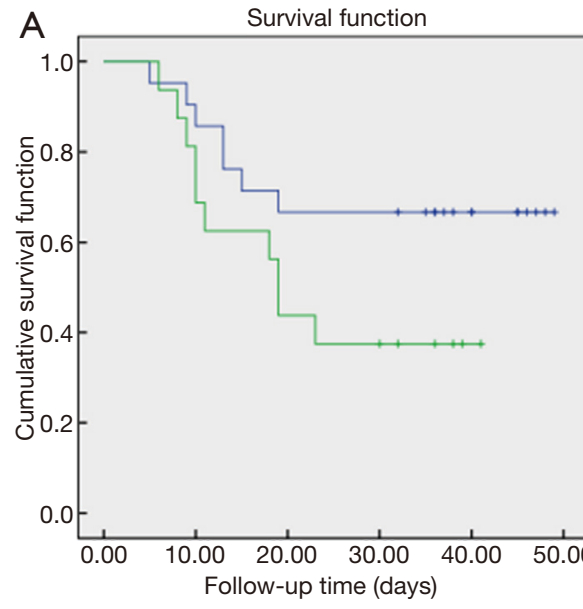

C

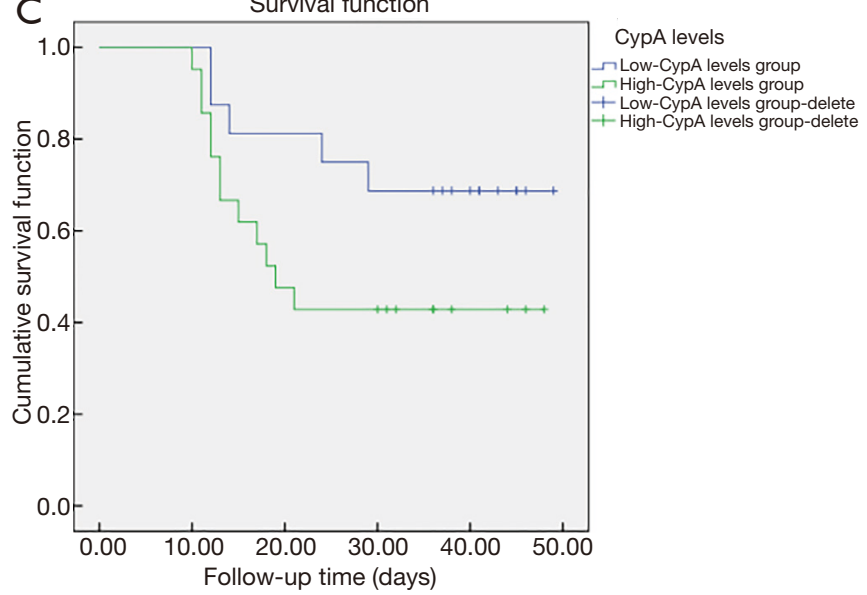

B

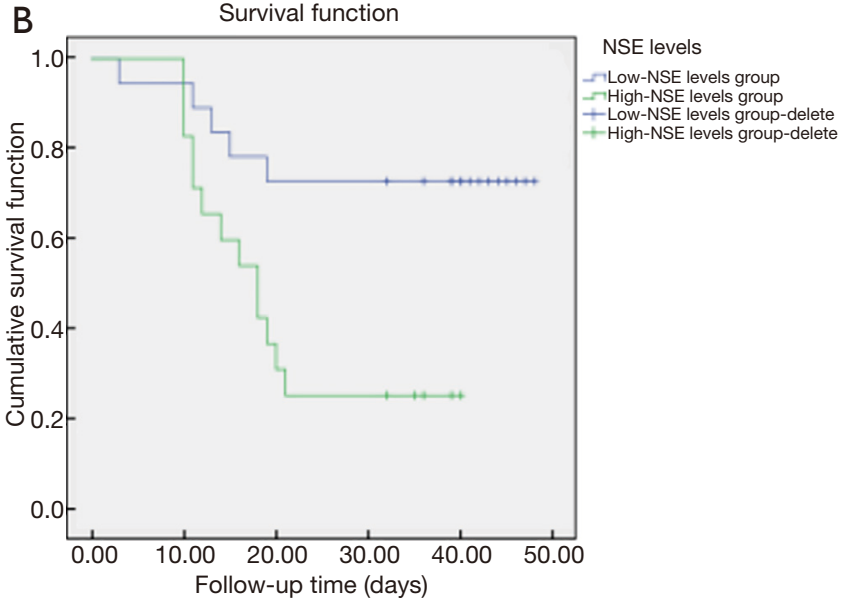

Figure 1 The relationship between S100B, NSE, and CypA levels on the prognosis and survival of patients. S100B, S100 calcium binding protein B; NSE, neuron-specific enolase; CypA, cyclophilin A.

of disability and death (12). Delirium refers to a group of symptoms of organic mental disorders, including disturbances in consciousness, significant excitement, restlessness, and disturbance in perception. Clinical practice has found that patients with severe head injury are prone to diffuse brain changes after delirium $(13,14)$. The progress of basic research and clinical research in recent years has shown that interventions for such patients are extremely important. Dynamic observation and accurate and effective treatment must be adopted to reduce the mortality rate $(15,16)$.

The $\mathrm{S} 100 \mathrm{~B}$ protein is an acidic calcium-binding protein, which is mainly found in astrocytes and oligodendrocytes of the central nervous system, and Schwann cells of the peripheral nervous system. It is currently believed that due to the integrity of brain cells and the blood-brain barrier in normal people, the content of S100B in cerebrospinal fluid is extremely low (17). Brain injury can be accompanied by mechanical brain cell destruction and blood-brain barrier damage, resulting in S100B being released outside the cell and entering the blood circulation (18-20). However, the mechanism of S100B as a marker of damage or in neuroprotection related to the pathophysiology of craniocerebral injury is still unclear. Therefore, this study analyzed the expression of the $\mathrm{S} 100 \mathrm{~B}$ protein in patients who were transferred from the ICU with severe craniocerebral injury and delirium. The levels of S100B have been shown to increase after craniocerebral injury and then decrease quickly, indicating an improvement in the patients' condition, then levels increase again or continue to increase, indicating that secondary injury or primary injury was aggravated (21). Furthermore, after 2 weeks of IFN- $\alpha$ therapy, S100B concentrations were shown to be higher in individuals with increased symptoms of depression, but the difference was not statistically significant (22). The variation 
in S100B concentration was mainly dependent on the degree of blood-brain barrier destruction and the severity and scope of brain injury. In this study, Kaplan-Meier survival curves demonstrated the relationship between S100B expression levels and patient prognosis, and the results showed that the survival time of the high expression group was shorter than that of the low expression group. It further showed that determining S100B levels could reflect the severity of head injury and predict the progression of the disease.

NSE belongs to the $\gamma \gamma$ subtype of the 5 enolase isoenzymes $\alpha \alpha, \beta \beta, \gamma \gamma, \alpha \beta$, and $\alpha \gamma$ that have been discovered. It is ubiquitous in the glycolytic metabolism of organisms, and can catalyze the conversion of $\alpha$-glycerol phosphate to phosphoenolpyruvate $(23,24)$. NSE has been proven to provide quantitative measurement of brain injury and/or ameliorate the diagnosis and outcome evaluation of ischemic stroke, cerebral hemorrhage, epilepsy, traumatic brain injury and coma patients after cardiopulmonary resuscitation (25). In another study, the change in NSE serum levels over time was different in patients with primary cortical contusion, diffuse axonal injury, and cerebral edema without local mass effect, suggesting that dynamic monitoring of NSE levels may help reflect the different physiological and pathological conditions after head injury (26). In the dexmedetomidine group, the median values of brain-derived neurotrophic factor (BDNF), NSE and S100B were significantly lower on the day of the diagnosis of delirium and the third day after the diagnosis of delirium. The incidence of irritation in the dexmedetomidine group was significantly lower than that in the normal group (27). The results of this study showed that the NSE level of the research group was higher than that of control group, and the survival time of the NSE high expression group was much lower than that of the low expression group. These results suggested that the determination of serum NSE levels could help evaluate the prognosis of patients with severe head injuries combined with delirium and multiple injuries.

CypA is the earliest discovered and most widely studied cyclophilin, which has a variety of biological functions. Recent studies have shown that CypA is also an intracellular receptor for cyclosporin, which can modulate cyclosporin to exert immunosuppressive effects, thereby regulating the body's immune function and inflammatory response $(28,29)$. Several studies have implicated CypA as a mediator of the ApoE4 pathway that predisposes patients to Alzheimer's disease. Elevated CypA concentrations in pericytes cause nuclear translocation of NF-kB and increase the activation of matrix metalloproteinase-9 (MMP-9) (30). In this study, the serum CypA levels of patients in the research group were significantly higher than those of the control group, suggesting that traumatic brain injury could cause the brain tissue to release a large amount of CypA, which might participate in the secondary inflammatory response. In the logistic regression model and the Kaplan-Meier survival curves, it was found that the survival time of the group with elevated CypA levels was significantly reduced, and the abnormal increase in levels was an independent risk factor that affected the prognosis of patients with severe head injury and delirium who were transferred out of the ICU. These results indicated that serum CypA had a strong predictive ability for the prognosis of ICU patients with severe head injury and delirium combined with multiple injuries.

In summary, the serum levels of S100B, NSE, and CypA are closely related to the prognosis of patients with delirium transferred out of the ICU with severe craniocerebral injury. They can be used as molecular markers for predicting the prognosis of patients, and may serve as potential targets for treatment.

\section{Acknowledgments}

Funding: None.

\section{Footnote}

Reporting Checklist: The authors have completed the STROBE reporting checklist. Available at http://dx.doi. org/10.21037/apm-21-424

Data Sharing Statement: Available at http://dx.doi. org/10.21037/apm-21-424

Conflicts of Interest: All authors have completed the ICMJE uniform disclosure form (available at http://dx.doi. org/10.21037/apm-21-424). The authors have no conflicts of interest to declare.

Ethical Statement: The authors are accountable for all aspects of the work in ensuring that questions related to the accuracy or integrity of any part of the work are appropriately investigated and resolved. The study was conducted in accordance with the Declaration of Helsinki (as revised in 2013). This study was approved by the Sichuan Provincial People's Hospital, University of Electronic 
Science and Technology of China (No. 20171208). All patients agreed to participate in this study and signed an informed consent form.

Open Access Statement: This is an Open Access article distributed in accordance with the Creative Commons Attribution-NonCommercial-NoDerivs 4.0 International License (CC BY-NC-ND 4.0), which permits the noncommercial replication and distribution of the article with the strict proviso that no changes or edits are made and the original work is properly cited (including links to both the formal publication through the relevant DOI and the license). See: https://creativecommons.org/licenses/by-nc-nd/4.0/.

\section{References}

1. Samuels JM, Moore EE, Silliman CC, et al. Severe traumatic brain injury is associated with a unique coagulopathy phenotype. J Trauma Acute Care Surg 2019;86:686-93.

2. Picetti E, Rossi S, Abu-Zidan FM, et al. WSES consensus conference guidelines: monitoring and management of severe adult traumatic brain injury patients with polytrauma in the first 24 hours. World J Emerg Surg 2019;14:53.

3. Watanabe T, Kawai Y, Iwamura A, et al. Outcomes after Traumatic Brain Injury with Concomitant Severe Extracranial Injuries. Neurol Med Chir (Tokyo) 2018;58:393-9.

4. Ganau M, Lavinio A, Prisco L. Delirium and agitation in traumatic brain injury patients: an update on pathological hypotheses and treatment options. Minerva Anestesiol 2018;84:632-40.

5. Intensive therapy in patients with severe traumatic brain injury complicated by delirium. Anesteziol Reanimatol 2012:48-51.

6. Abboud T, Mende KC, Jung R, et al. Prognostic Value of Early S100 Calcium Binding Protein B and NeuronSpecific Enolase in Patients with Poor-Grade Aneurysmal Subarachnoid Hemorrhage: A Pilot Study. World Neurosurg 2017;108:669-75.

7. Hirai H. Production of the neuron-specific enolase in astrocytes, but not in neurons, under the neuronal inflammation. Seikagaku 2017;89:241-3.

8. Xue C, Sowden MP, Berk BC. Extracellular and Intracellular Cyclophilin A, Native and PostTranslationally Modified, Show Diverse and Specific Pathological Roles in Diseases. Arterioscler ThrombVasc
Biol 2018;38:986-93.

9. Jin HH, Mao TM, Bai ZQ, et al. The 90-day prognostic value of serum cyclophilin A in traumatic brain injury. Clin Chim Acta 2018;484:258-64.

10. Lee J, Cho Y, Choi KS, et al. Efficacy and safety of erythropoietin in patients with traumatic brain injury: A systematic review and meta-analysis. Am J Emerg Med 2019;37:1101-7.

11. Khurrum M, Chehab M, Joseph B. Non-Neurological Organ Dysfunction in Severe Isolated Traumatic Brain Injury. J Trauma Acute Care Surg 2020;89:405-10.

12. Trimmel H, Herzer G, Schöchl H, et al. Intensive care treatment of traumatic brain injury in multiple trauma patients : Decision making for complex pathophysiology. Unfallchirurg 2017;120:739-44.

13. Mattison MLP. Delirium. Ann Intern Med 2020;173:ITC49-ITC64.

14. Pařízková R. Delirium in the intensive care unit. Vnitr Lek 2019;65:433-9.

15. Rieck KM, Pagali S, Miller DM. Delirium in hospitalized older adults. Hosp Pract (1995) 2020;48:3-16.

16. Lauretani F, Bellelli G, Pelà G, et al. Treatment of Delirium in Older Persons: What We Should Not Do! Int J Mol Sci 2020;21:2397.

17. Koh SX, Lee JK. S100B as a marker for brain damage and blood-brain barrier disruption following exercise. Sports Med 2014;44:369-85.

18. Dietrich Mde O, Souza DO, Portela LV. Serum S100B protein: what does it mean during exercise? Clin J Sport Med 2004;14:368; author reply 368-9.

19. Schulte S, Schiffer T, Sperlich B, et al. Response to the Letter to the Editor of Sorci et al. Causes of elevated serum levels of S100B protein in athletes. Eur J Appl Physiol 2013;113:821-2.

20. Donato R, Riuzzi F, Sorci G. Causes of elevated serum levels of S100B protein in athletes. Eur J Appl Physiol 2013;113:819-20.

21. Liu S, Zhang Y, Zhao Y, et al. Effects of hypothermia on S100B and glial fibrillary acidic protein in asphyxia rats after cardiopulmonary resuscitation. Cell Biochem Biophys 2015;71:401-6.

22. Cicek IE, Cicek E, Kayhan F, et al. The roles of BDNF, S100B, and oxidative stress in interferon-induced depression and the effect of antidepressant treatment in patients with chronic viral hepatitis: a prospective study. J Psychosom Res 2014;76:227-32.

23. Anastasiades KD, Mullins RE, Conn RB. Neuron-specific enolase. Assessment by ELISA in patients with small cell 
carcinoma of the lung. Am J Clin Pathol 1987;87:245-9.

24. Viallard JL, Ven Murthy MR, Dastugue B. Rapid electrophoretic determination of neuron-specific enolase isoenzymes in serum. Clin Chem 1986;32:593-7.

25. Isgrò MA, Bottoni P, Scatena R. Neuron-Specific Enolase as a Biomarker: Biochemical and Clinical Aspects. Adv Exp Med Biol 2015;867:125-43.

26. Toupalík P, Klír P, Bouska I, et al. Immunohistochemical methods in the differential diagnosis of primary traumatic and subsequent secondary cerebral changes. Soud Lek 2000;45:18-21.

27. Li Y, Yu ZX, Ji MS, et al. A Pilot Study of the Use of Dexmedetomidine for the Control of Delirium by Reducing the Serum Concentrations of Brain-Derived
Neurotrophic Factor, Neuron-Specific Enolase, and S100B in Polytrauma Patients. J Intensive Care Med 2019;34:674-81.

28. A Abdullah A, Abdullah R, A Nazariah Z, et al. Cyclophilin $A$ as a target in the treatment of cytomegalovirus infections. Antivir Chem Chemother. 2018;26:2040206618811413.

29. Garimella V, McVoy JS, Oh U. The contribution of cyclophilin A to immune-mediated central nervous system inflammation. J Neuroimmunol 2020;339:577118.

30. Bell RD, Winkler EA, Singh I, et al. Apolipoprotein $\mathrm{E}$ controls cerebrovascular integrity via cyclophilin A. Nature 2012;485:512-6.

(English Language Editor: C. Betlazar-Maseh)
Cite this article as: Gao Y, Duan J, Ji H, Lu W. Levels of S100 calcium binding protein B (S100B), neuron-specific enolase (NSE), and cyclophilin A (CypA) in the serum of patients with severe craniocerebral injury and multiple injuries combined with delirium transferred from the ICU and their prognostic value for its prognosis. Ann Palliat Med 2021;10(3):3371-3378. doi: 10.21037/apm-21-424 